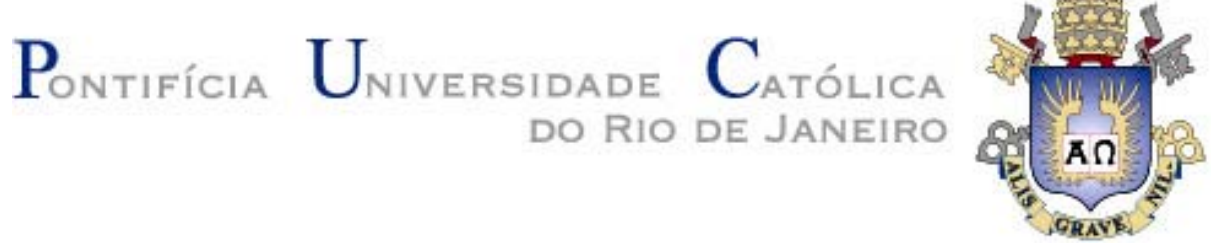

Shelly Cristiane D'Avila Medeiros Algoritmos Genéticos

Dissertação apresentada ao Programa de PósGraduação em Engenharia Elétrica da PUC-Rio como requisito parcial para obtenção do título de Mestre em Engenharia Elétrica.

Orientador: Marco Aurélio C. Pacheco

Rio de Janeiro Novembro de 2005 


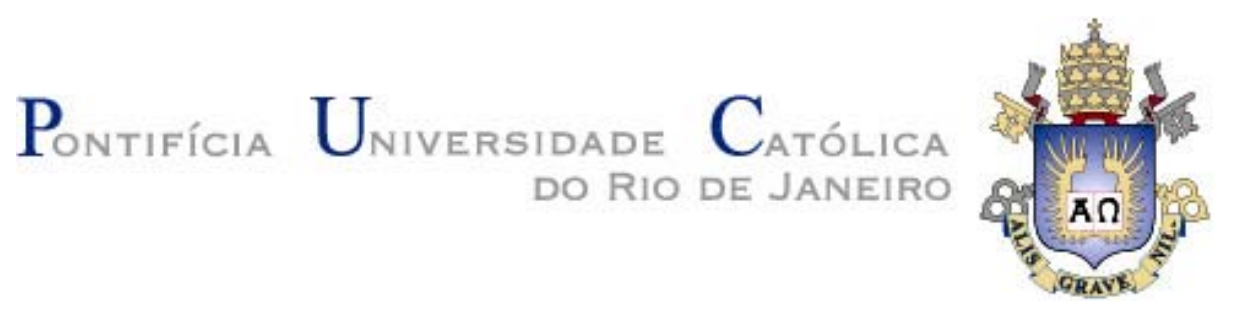

Shelly Cristiane D'Ávila Medeiros

\title{
Inversão de Parâmetros em Dados Sísmicos por \\ Algoritmos Genéticos
}

\begin{abstract}
Dissertação apresentada como requisito parcial para obtenção do grau de Mestre pelo Programa de PósGraduação em Engenharia Elétrica do Departamento de Engenharia Elétrica do Centro Técnico Científico da PUCRio. Aprovada pela Comissão Examinadora abaixo assinada.
\end{abstract}

\author{
Prof. Marco Aurélio Cavalcanti Pacheco \\ Orientador \\ Departamento de Engenharia Elétrica - PUC-Rio \\ Prof. Carlos Roberto Hall Barbosa \\ Departamento de Engenharia Elétrica - PUC-Rio
}

Prof. Sergio Augusto Barreto da Fontoura

Departamento de Engenharia Civil - PUC-Rio

Prof. Eduardo Lopes de Faria CENPES/PETROBRÁS

Prof. José Eugenio Leal

Coordenador Setorial do Centro

Técnico Científico - PUC-Rio

Rio de Janeiro, 21 de novembro de 2005 
Todos os direitos reservados. É proibida a reprodução total ou parcial do trabalho sem autorização da universidade, da autora e do orientador.

\section{Shelly Cristiane D'Ávila Medeiros}

Graduou-se em Bacharel em Informática na Pontifícia Universidade Católica do Rio Grande do Sul em 2001. Suas principais áreas de atuação são Engenharia de Petróleo e Análise de Sistemas.

Ficha Catalográfica

Medeiros, Shelly Cristiane D'Avila

Inversão de parâmetros em dados sísmicos por algoritmos genéticos / Shelly Cristiane D'Avila Medeiros; orientador: Marco Aurélio C. Pacheco. - Rio de Janeiro: PUC, Departamento de Engenharia Elétrica, 2005.

89 f. : il. ; $30 \mathrm{~cm}$

Dissertação (mestrado) - Pontifícia Universidade Católica do Rio de Janeiro, Departamento de Engenharia Elétrica.

Inclui referências bibliográficas.

1. Engenharia elétrica - Teses. 2. Algoritmos Genéticos. 3. Inversão Sísmica. 4. Inversão de Parâmetros Físicos. 5. Análise de Velocidade. 6. Velocidade root mean square. I. Pacheco, Marco Aurélio C. II. Pontifícia Universidade Católica do Rio de Janeiro. Departamento de Engenharia Elétrica. III. Título.

CDD: 621.3 


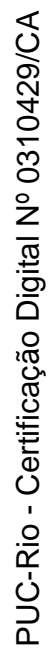

Para meus amores, Fabrício, Ismael \& Rita e Geraldo \& Luzia. 
Tudo tem a sua ocasião própria, e há tempo para todo propósito debaixo do céu. Há tempo de nascer, e tempo de morrer; tempo de plantar, e tempo de arrancar o que se plantou; tempo de matar, e tempo de curar; tempo de derribar, e tempo de edificar; tempo de chorar, e tempo de rir; tempo de prantear, e tempo de dançar; tempo de espalhar pedras, e tempo de ajuntar pedras; tempo de abraçar, e tempo de absterse de abraçar; tempo de buscar, e tempo de perder; tempo de guardar, e tempo de deitar fora; tempo de rasgar, e tempo de coser; tempo de estar calado, e tempo de falar; tempo de amar, e tempo de odiar; tempo de guerra, e tempo de paz.

Eclesiastes 3:1-8

Vi que também isso vem da mão de Deus.

Eclesiastes 2:24 


\section{Resumo}

D'Avila Medeiros, Shelly Cristiane. Inversão de Parâmetros em Dados Sísmicos por Algoritmos Genéticos. Rio de Janeiro, 2005. 89p. Dissertação de Mestrado - Departamento de Engenharia Elétrica, Pontifícia Universidade Católica do Rio de Janeiro.

Esta dissertação investiga o uso de Algoritmos Genéticos aplicados em dados sísmicos com o objetivo de obter parâmetros físicos e atributos sísmicos que auxiliem na caracterização das rochas de um subsolo terrestre. Os dados sísmicos têm sido extensamente empregados no setor de exploração de petróleo. As aplicações envolvendo sísmica não se restringem na busca por novas reservas de petróleo, mas também são usadas para projetar novos poços e melhorar a produção dos reservatórios de petróleo. O levantamento de dados sísmicos permite analisar extensas áreas da subsuperfície com custo praticável em relação a outras técnicas. Entretanto, a interpretação desses dados com o objetivo de obter informações relevantes e acuradas não é uma tarefa simples. Para isto, várias técnicas de inversão sísmica vêm sendo desenvolvidas. Este trabalho consistiu em avaliar uma alternativa que emprega Algoritmos Genéticos para inverter parâmetros a partir de dados sísmicos. Existem 3 etapas principais neste trabalho. Primeiramente, foram estudados o tema da exploração sísmica e a técnica de Algoritmos Genéticos. Na segunda etapa foi definido um modelo, usando Algoritmos Genéticos, que busca, neste caso, minimizar uma medida de erro, para obtenção dos parâmetros objetivos. Finalmente, foi implementado um sistema a partir do modelo proposto e realizados os estudos de casos com dados sísmicos sintéticos para avaliar o seu desempenho. O modelo baseado em Algoritmos Genéticos foi avaliado submetendo-se seus resultados a um especialista e comparando-os com os da busca aleatória. Os resultados obtidos se mostraram consistentemente satisfatórios e sempre superiores aos da busca exaustiva.

\section{Palavras-chave}

Algoritmos Genéticos; Inversão Sísmica; Inversão de Parâmetros Físicos; Análise de Velocidade; Velocidade root mean square 


\section{Abstract}

D'Avila Medeiros, Shelly Cristiane. Inversion of Parameters in Seismic Data by Genetic Algorithms. Rio de Janeiro, 2005. 89p. Dissertação de Mestrado - Departamento de Engenharia Elétrica, Pontifícia Universidade Católica do Rio de Janeiro.

This dissertation investigates the use of Genetic Algorithms applied to seismic data with the objective of obtaining physical parameters and seismic attributes that would facilitate the characterization of rocks in terrestrial subsoil. The seismic data has been extensively utilized in the field of petroleum exploration. The applications involving seismic are not restrained to the search for new petroleum reserves, but are also used to project new wells and to improve the production of existing petroleum reservoirs. The survey of seismic data allows the analysis of extended areas of the subsurface at an affordable price relative to other techniques. However, the interpretation of the data with the objective of obtaining relevant and accurate information is not an easy task. For that, several seismic inversion techniques are being developed. This work consists in evaluating an alternative that uses Genetic Algorithms to invert parameters from seismic data. There are 3 main stages in this work. Initially, the theme of seismic exploration and the technique of Genetic Algorithms have been studied. On the second stage a model has been defined, using Genetic Algorithms, which aims, in this case, to minimize an error measurement, obtaining objective parameters. Finally, a system from the proposed model has been implanted and the study of cases with synthetic seismic data has been executed to evaluate its performance. The process of optimizing has been compared to the process of random search and the results obtained by the model have always been superior.

\section{Keywords}

Genetic Algorithms; Seismic inversion; Inversion of physical parameters; Velocity analysis; root mean square velocity. 


\section{Sumário}

1 Introdução 16

1.1. Motivação 16

1.2. Objetivos do Trabalho 18

1.3. Descrição do Trabalho 19

1.4. Organização da Dissertação 20

2 Exploração Sísmica

2.1. Sísmica de Reflexão 22

2.2. Sismograma 25

2.3. Ponto Médio Comum (CMP - Common-midpoint) 26

2.4. Velocidades Sísmicas 28

2.5. Aquisição Sísmica Sintética 29

2.5.1. ANRAY 31

2.5.2. CREWES 31

2.6. Inversão Sísmica 32

3 Algoritmos Genéticos 33

3.1. Componentes de Algoritmos Genéticos 33

3.2. Representação 35

3.3. Codificação e Decodificação 35

3.4. Avaliação 35

3.5. Operadores Genéticos 36

3.6. Parâmetros da Evolução 37

4 Sistema de Identificação de Parâmetros em Dados Sísmicos por Algoritmos Genéticos 39

4.1. Arquitetura do Modelo Proposto 39

4.1.1. Módulo 1 - Algoritmo Genético 40

4.1.2. Módulo 2 - Geração de Dados Sísmicos Sintéticos 41

4.1.3. Módulo 3 - Seleção de Tempos de Trânsito 42

4.1.4. Módulo 4 - Cálculo da Medida de Erro 44 
4.2. Algoritmo Genético Proposto 48

4.2.1. Estrutura do Cromossomo 48

4.2.2. Operadores Genéticos $\quad 52$

4.2.3. Decodificação 53

4.3. O Simulador $\quad 55$

5 Estudo de Casos $\quad 57$

5.1. Estudo de Caso 1: Velocidade Intervalar e Espessura da Camada 57

5.1.1. Design

5.1.2. Resultados $\quad 59$

5.2. Estudo de Caso 2.a: Velocidade Root Mean Square e Tempo para Incidência Normal - Dados Sintéticos 67

5.2.1. Design 68

5.2.2. Resultados $\quad 69$

5.3. Estudo de Caso 3: Velocidade Intervalar, Densidade, Espessura da Camada e Número de Eventos $\quad 76$

$\begin{array}{ll}\text { 5.3.1. Design } & 76\end{array}$

5.3.2. Resultados $\quad 79$

5.4. Estudo de Caso 2.b: Velocidade Root Mean Square e Tempo para Incidência Normal - Dados Reais 81

5.4.1. Design $\quad 81$

5.4.2. Resultados 83

6 Conclusões e Trabalhos Futuros $\quad 86$

7 Referências Bibliográficas $\quad 88$ 


\section{Lista de figuras}

Figura 1 - Uma frente de ondas é gerada na subsuperfície por uma fonte e se propaga nas camadas inferiores. Ao encontrar uma interface parte da onda se reflete e parte se refrata. A onda refletida retorna à subsuperfície e os receptores capturam sua chegada. O tempo percorrido pelo pulso sísmico da fonte até os receptores e suas amplitudes na chegada são armazenados para estudos futuros.

Figura 2 - Movimento das partículas durante a passagem de uma onda. (a) onda P; (b) onda S (extraído de Sherrif \& Geldart ,1995).

Figura 3 - Esquema de partição de energia sísmica. Geração de ondas P e S refletidas e refratadas a partir de uma onda $P$ incidente numa interface plana.

Figura 4 - Representação gráfica de um sismograma composto por 4 traços sísmicos e 1 evento sísmico. O evento corresponde à curva semelhante a uma semi-hipérbole representada pela linha azul.

Figura 5 - Aquisição de dados sísmicos considerando uma fonte e vários receptores - uma única leitura para cada ponto de reflexão na interface. 27

Figura 6 - Aquisição de dados sísmicos considerando várias fontes e vários receptores - várias leituras para um mesmo ponto de reflexão na interface.

Figura 7 - Velocidade média das camadas. 28

Figura 8 - Velocidade nmo. A figura (a) mostra o evento sísmico antes da correção. A figura (b) mostra o evento sísmico corrigido com o termo $\Delta t_{N M O}$ (extraído de Yilmaz, 2001).

Figura 9 - Para a camada $\mathrm{N}$ é calculada a velocidade rms, considerando que a reflexão da onda recebida pelo receptor $\mathrm{R}$ foi no ponto $\mathrm{D}$ desta camada. 29

Figura 10 - Procedimento básico do algoritmo genético 34

Figura 11 - Cruzamento de um ponto 36

Figura 12 - Mutação $\quad 37$

Figura 13 - Descrição do sistema de inversão de parâmetros por Algoritmos Genéticos a partir de dados sísmicos.

Figura 14 - Exemplo de modelo da subsuperfície com $n$ camadas horizontais homogêneas e isotrópicas. 
Figura 15 - Estrutura do cromossomo para o primeiro estudo de caso. Este cromossomo representa a velocidade intervalar e a espessura das camadas, parâmetros estes separados em dois segmentos. Cada posição i dos segmentos corresponde a uma camada do modelo.

Figura 16 - Estrutura do cromossomo para o segundo estudo de caso. Este cromossomo representa a velocidade rms e o tempo para incidência normal das camadas, parâmetros estes separados em dois segmentos. Cada posição i dos segmentos corresponde a uma camada do modelo.

Figura 17 - Estrutura do cromossomo para o terceiro estudo de caso. Este cromossomo representa a velocidade intervalar, a densidade e a espessura das camadas, parâmetros estes separados em três segmentos. O segmento 1 deste cromossomo representa a máscara de ativação. Cada posição i dos segmentos corresponde a uma camada do modelo.

Figura 18 - Sismograma de referência.

Figura 19 - Estudo de caso 1: Modelo da subsuperfície com 2 camadas horizontais, homogêneas e isotrópicas.

Figura 20 - Eventos selecionados a partir do sismograma de referência.

Figura 21 - Rodada com a seguinte configuração do AG: Tx. Mut 0.001, Tx. Cruz. 0.7, Intv. Ger. 0.3, nº gerações 300.

Figura 22 - Rodada com a seguinte configuração do AG: Tx. Mut 0.01, Tx. Cruz. 0.7, Intv. Ger. 0.3, $\mathrm{n}^{\circ}$ gerações 300.

Figura 23 - Rodada com a seguinte configuração do AG: Tx. Mut 0.1, Tx. Cruz.

0.7, Intv. Ger. 0.3, $\mathrm{n}^{\circ}$ gerações 300.

Figura 24 - Rodada com a seguinte configuração do AG: Tx. Mut 0.2, Tx. Cruz. 0.7, Intv. Ger. 0.3, $n^{\circ}$ gerações 300.

Figura 25 - Rodada com a seguinte configuração do AG: Tx. Mut 0.3, Tx. Cruz. 0.7, Intv. Ger. 0.3, nº gerações 300.

Figura 26 - Rodada com a seguinte configuração do AG: Tx. Mut 0.4, Tx. Cruz.

0.7, Intv. Ger. 0.3, $\mathrm{n}^{0}$ gerações 300.

Figura 27 - Rodada com a seguinte configuração do AG: Tx. Mut 0.3, Tx. Cruz.

0.001, Intv. Ger. 0.3, $\mathrm{n}^{0}$ gerações 300.

Figura 28 - Rodada com a seguinte configuração do AG: Tx. Mut 0.3, Tx. Cruz. 0.01, Intv. Ger. 0.3, nº gerações 300.

Figura 29 - Rodada com a seguinte configuração do AG: Tx. Mut 0.3, Tx. Cruz. 0.1, Intv. Ger. 0.3, $\mathrm{n}^{0}$ gerações 300.

Figura 30 - Rodada com a seguinte configuração do AG: Tx. Mut 0.3, Tx. Cruz. 
0.2, Intv. Ger. 0.3, $\mathrm{n}^{\circ}$ gerações 300 .

Figura 31 - Rodada com a seguinte configuração do AG: Tx. Mut 0.3, Tx. Cruz.

0.8, Intv. Ger. 0.3, $\mathrm{n}^{\circ}$ gerações 300.

64

Figura 32 - Comparação entre a busca aleatória e a evolução por AG. A configuração do AG nesta rodada é composta por : Tx. Mut 0.3, Tx. Cruz. 0.8, Intv. Ger. 0.3, nº gerações 300.

Figura 33 - Comparação entre o sismograma de referência e os eventos gerados a partir de uma boa solução dada pelo AG com o valor de MAPE (fitness) de 1.4945.

Figura 34 - Comparação entre os eventos do sismograma de referência representados pelas linhas cheias em azul com os eventos gerados a partir de uma solução dada pelo AG representados pelas linhas tracejadas em vermelho. Valor do MAPE (fitness) de 0.9657.

Figura 35 - Sismograma de referência gerado sinteticamente. 68

Figura 36 - Estudo de caso 2.a: Modelo da subsuperfície com 2 camadas horizontais, homogêneas e isotrópicas.

Figura 37 - Eventos sísmicos selecionados a partir do sismograma de referência. Cada evento representa uma camada de rocha do modelo. 69

Figura 38 - Rodada com a seguinte configuração do AG: Tx. Mut 0.001, Tx. Cruz. 0.7, Intv. Ger. 0.3, nº gerações 600.

Figura 39- Rodada com a seguinte configuração do AG: Tx. Mut 0.01, Tx. Cruz. 0.7, Intv. Ger. 0.3, nº gerações 600.

Figura 40- Rodada com a seguinte configuração do AG: Tx. Mut 0.1, Tx. Cruz. 0.7, Intv. Ger. 0.3, $\mathrm{n}^{\circ}$ gerações 600 .

Figura 41- Rodada com a seguinte configuração do AG: Tx. Mut 0.2, Tx. Cruz.

$$
0.7 \text {, Intv. Ger. 0.3, } n^{\circ} \text { gerações } 600 .
$$

Figura 42- Rodada com a seguinte configuração do AG: Tx. Mut 0.3, Tx. Cruz. 0.7, Intv. Ger. 0.3, nº gerações 600 .

Figura 43 - Rodada com a seguinte configuração do AG: Tx. Mut 0.1, Tx. Cruz.

0.001 , Intv. Ger. 0.3, $n^{0}$ gerações 600.

Figura 44 - Rodada com a seguinte configuração do AG: Tx. Mut 0.1, Tx. Cruz.

0.01, Intv. Ger. 0.3, nº gerações 600.

Figura 45 - Rodada com a seguinte configuração do AG: Tx. Mut 0.1, Tx. Cruz. 0.1, Intv. Ger. 0.3, $\mathrm{n}^{0}$ gerações 600.

Figura 46 - Rodada com a seguinte configuração do AG: Tx. Mut 0.1, Tx. Cruz. 0.2, Intv. Ger. 0.3, nº gerações 600 . 
Figura 47 - Rodada com a seguinte configuração do AG: Tx. Mut 0.3, Tx. Cruz.

0.8, Intv. Ger. 0.3, $\mathrm{n}^{\circ}$ gerações 600.

Figura 48 - Rodada com a seguinte configuração do AG: Tx. Mut 0.3, Tx. Cruz.

0.9, Intv. Ger. 0.3, $\mathrm{n}^{\circ}$ gerações 600.

74

Figura 49 - Comparação entre a busca aleatória e a evolução por AG. A configuração do AG nesta rodada é composta por : Tx. Mut 0.3, Tx. Cruz. 0.9 , Intv. Ger. 0.3, $n^{\circ}$ gerações 600 .

75

Figura 50 - Comparação entre o sismograma de referência e os eventos gerados a partir do melhor indivíduo do AG com o valor de MAPE (fitness) de 0.19143.

Figura 51 - Exemplo de sismograma sintético gerado com o simulador crewes.

Figura 52 - Exemplo de sismograma sintético gerado com o simulador anray. 78 Figura 53 - Sismograma de referência gerado sinteticamente 79

Figura 54 - Estudo de caso 3: Modelo da subsuperfície com 2 camadas horizontais, homogêneas e isotrópicas.

Figura 55 - Comparação entre o sismograma de referência e os eventos gerados a partir do melhor indivíduo do AG com o valor de RMSE (fitness) de 0.00005 .

Figura 56 - Sismograma real (referência).

Tabela 21 - Parâmetros definidos para o sistema de aquisição no estudo de caso 2.b.

Figura 57 - Eventos sísmicos selecionados a partir do sismograma de referência. Cada evento representa uma camada de rocha do modelo. 83

Figura 58 - Estudo de caso 2.b: Modelo da subsuperfície com 2 camadas horizontais, homogêneas e isotrópicas.

83

Figura 59 - Comparação entre a busca aleatória e a evolução por AG. A configuração do AG nesta rodada é composta por : Tx. Mut 0.3, Tx. Cruz. 0.7, Intv. Ger. 0.3, nº gerações 600.

Figura 60 - Comparação entre o sismograma de referência real e os eventos gerados a partir de uma boa solução dada pelo AG com o valor de MAPE (fitness) de 0.35188 . 


\section{Lista de tabelas}

Tabela 1 - Exemplo de um sismograma contendo as amplitudes lidas nos receptores em cada instante de tempo. No tempo $\mathbf{t t}_{1}$ o receptor rec $_{1}$ está iniciando a leitura de uma onda e a amplitude lida neste tempo é 0.06. Nenhuma onda está retornando para os demais receptores no instante de tempo $\mathbf{t t}_{1}$.

Tabela 2 - Parâmetros que caracterizam as camadas de um modelo da subsuperfície. A camada de índice 1 tem velocidade intervalar $\mathrm{V}_{\mathrm{i} 1}$ e espessura $e_{1}$.

Tabela 3 - Exemplo de um sismograma contendo as amplitudes lidas nos receptores em cada instante de tempo. No tempo $\mathbf{t t}_{1}$ o receptor rec $_{1}$ está iniciando a leitura de uma onda e a amplitude lida neste tempo é 0.06. Nenhuma onda está retornando para os demais receptores no instante de tempo $\mathbf{t t}_{1}$. As células em cinza escuro contêm as amplitudes máximas de cada onda lida.

Tabela 4 - Conjunto de tempos de trânsito obtidos a partir de um sismograma por um processo de extração de características.

Tabela 5 - Exemplo de um conjunto de dados sísmicos objetivo. A célula cinza escuro representa o tempo de trânsito $\mathrm{tt}_{11}$, tempo de viagem da fonte ao receptor $\mathbf{r e c}_{1}$, de uma onda refletida na camada $\mathbf{c m d}_{1}$.

Tabela 6 - Exemplo de um conjunto de dados sísmicos gerados. Estes tempos de trânsito foram calculados a partir de um modelo da subsuperfície dado por um indivíduo decodificado do AG.

Tabela 7 - Sismograma de referência. As linhas da matriz indicam os índices dos instantes de tempo, já as colunas indicam os índices dos receptores. O conteúdo é formado pelos valores de amplitudes das ondas lidas.

Tabela 8 - Sismograma gerado a partir de um modelo da subsuperfície - meio definido por um indivíduo decodificado do AG.

Tabela 9 - Definição dos intervalos de valores mínimo e máximo para cada parâmetro a ser otimizado.

Tabela 10 - Definição dos intervalos de valores mínimo e máximo para cada parâmetro a ser otimizado.

51

Tabela 11 - Definição dos intervalos de valores mínimo e máximo para cada 
parâmetro a ser otimizado.

Tabela 12 - Parâmetros definidos para o sistema de aquisição no estudo de caso 1.

Tabela 13 - Resultados para o estudo de caso 1. Cada parâmetro resultante é obtido a partir do cálculo da média dos melhores indivíduos em 10 experimentos. As células em cinza claro mostram os parâmetros do AG que foram alterados, enquanto os demais parâmetros permaneceram fixos. A linha em que as células estão em cinza escuro representa a melhor configuração obtida para o AG.

Tabela 15 - Parâmetros do modelo da subsuperfície obtidos a partir de uma solução dada pelo AG.

Tabela 16 - Parâmetros definidos para o sistema de aquisição no estudo de caso 2.a.

Tabela 17 - Resultados para o estudo de caso 2. O valor de erro MAPE é obtido a partir do cálculo da média dos melhores indivíduos em 10 experimentos. As células em cinza claro mostram os parâmetros do $A G$ que foram alterados, enquanto os demais parâmetros permaneceram fixos. A linha em que as células estão em cinza escuro representa a melhor configuração obtida para o AG.

Tabela 18 - Parâmetros do modelo da subsuperfície obtidos com o melhor indivíduo do AG com a seguinte configuração: Tx. Mut 0.3, Tx. Cruz. 0.7, Intv. Ger. 0.3, $n^{\circ}$ gerações 600 .

Tabela 19 - Parâmetros definidos para o sistema de aquisição no estudo de caso 3. 